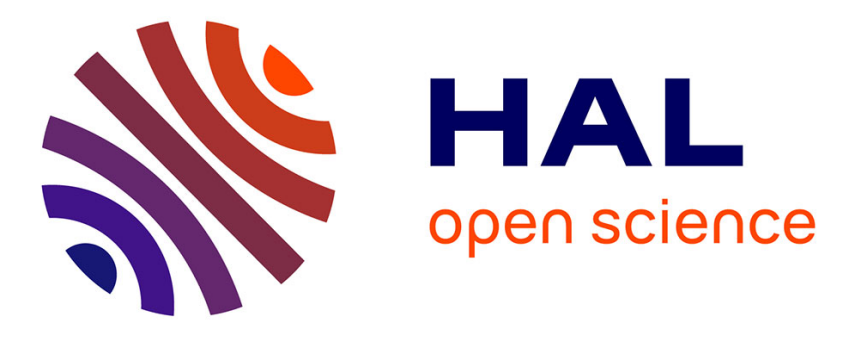

\title{
Understanding and overcoming horizontal separation complexity in air traffic control: an expert/novice comparison
}

Nicolas Durand, Jean-Baptiste Gotteland, Nadine Matton, Léa Bortolotti, Margot Sandt

\section{To cite this version:}

Nicolas Durand, Jean-Baptiste Gotteland, Nadine Matton, Léa Bortolotti, Margot Sandt. Understanding and overcoming horizontal separation complexity in air traffic control: an expert/novice comparison. Cognition, Technology and Work, 2021, 23, pp.481-496. 10.1007/s10111-020-00634-z . hal-02643660

\section{HAL Id: hal-02643660 \\ https://hal-enac.archives-ouvertes.fr/hal-02643660}

Submitted on 30 Jun 2020

HAL is a multi-disciplinary open access archive for the deposit and dissemination of scientific research documents, whether they are published or not. The documents may come from teaching and research institutions in France or abroad, or from public or private research centers.
L'archive ouverte pluridisciplinaire HAL, est destinée au dépôt et à la diffusion de documents scientifiques de niveau recherche, publiés ou non, émanant des établissements d'enseignement et de recherche français ou étrangers, des laboratoires publics ou privés. 


\title{
Understanding and Overcoming Horizontal Separation Complexity in Air Traffic Control: An expert/novice comparison
}

\author{
Nicolas Durand · Jean-Baptiste Gotteland . \\ Nadine Matton · Léa Bortolotti · Margot Sandt
}

Received: date / Accepted: date

Keywords: conflict detection, decision support tool, visualization tool, air traffic complexity, air traffic control

\author{
Abstract \\ N. Durand \\ ENAC, 7 av Ed Belin 31055 Toulouse \\ Tel.: +33-562259565 \\ E-mail: nicolas.durand@enac.fr \\ J.B. Gotteland \\ ENAC, 7 av Ed Belin 31055 Toulouse \\ Tel.: +33-562259584 \\ E-mail: jean-baptiste.gotteland@enac.fr \\ N. Matton \\ ENAC, 7 av Ed Belin 31055 Toulouse \\ Tel.: +33-562259618 \\ E-mail: nadine.matton@enac.fr \\ L. Bortolotti \\ DGAC \\ E-mail: lea.bortolotti@aviation-civile.gouv.fr \\ M. Sandt \\ DGAC \\ E-mail: margot.sandt@aviation-civile.gouv.fr
}

Humans still play a key role in air traffic control but their performances limit the capacity of the airspace and are responsible for delays. At the tactical level, even though Air Traffic Controllers (ATCO) are trained for years, their performances are limited. In this article, we first isolated the tactical horizontal deconfliction task and explained its mathematical complexity. We observed through a simple experiment conducted on 
trainee and experienced ATCOs its complexity on random traffic in a part-task trainer displaying two to five aircraft trajectories at the same altitude. We compared performances of trainee ATCOs with experienced ATCOs using two different displays: a basic display showing information on aircraft positions and a dynamic visualization tool that represents the conflicting portions of aircraft trajectories and the evolution of the conflict zone when the user adds a maneuver to an aircraft. The tool allows the user to dynamically check the potential conflicting zones with the computer mouse before making a maneuver decision. Results showed that in easy situations (two aircraft), performance was similar with both displays and groups. However, as the complexity of the situations grows (from three to five aircraft), the dynamic visualization tool enables users to solve the conflicts more efficiently. Using the tool leads to fewer unsolved conflicts. Even if experienced ATCOs performed much better than trainee ATCOs on complex situations, they also performed much better with the conflict visualization tool than without on such situations.

\section{Introduction}

Air Traffic Controller tasks are diverse and complex. At the tactical level, they deal with many different tasks in order to keep aircraft separated by a separation standard of five nautical miles in the horizontal plane or 1000 feet in the vertical plane. They supervise the traffic inside a space area called control sector and give simple instructions to pilots, such as change heading, change level, go direct to some point. They can also stop the climb of an aircraft, anticipate its descent, and change its speed when it is possible. One important task is to detect and solve conflicts between two or more aircraft.

New technologies offer opportunities to develop tools that can replace controllers in some difficult tasks. A lot of research has been done on conflict resolution automation [Durand et al (1996); Oh et al (1997); Frazzoli et al (2001); Pallottino et al (2002); Christodoulou and Kontogeorgou (2008); Alaeddini et al (2011); Gariel and Feron (2009); Allignol et al (2013)]. Mathematical models proposed by the literature are more or less realistic : many models do not take uncertainties into account and are not realistic enough to consider a future implementation, but even with the most realistic models using basic maneuvers [Durand et al (1996); Allignol et al (2013)], the solutions proposed by automatic solvers on complex situations are difficult to understand by humans and could certainly not be easily used as an aid for air traffic controllers. This is not due to the weaknesses of the model that can take many realistic uncertainties into account but to the complexity of the optimal solution itself for conflicts involving many aircraft. This is why, in this article, we try to focus on a tool, taking into account speed and heading change uncertainties, that can help ATCOs build their own solution.

This article debates the following statements:

- The horizontal conflict resolution problem is mathematically very complex.

- Even when this task is isolated and limited to a single cluster of five aircraft, experienced ATCOs have difficulties solving it. 
- A dynamic visualization of conflicts zones can help ATCOs dealing with this complexity. This visualization does not directly give a global solution but helps ATCOs to solving complex conflicts.

We first show that the mathematical underlying complexity is high and might explain why even experienced ATCOs cannot deal easily with five aircraft conflicts when solutions exist, and this is the case even when this specific task is isolated. We show that a simple dynamic conflict visualization tool can ease the resolution task especially for experienced ATCOs. We used random traffic examples in order to limit the use of recognition skills by experienced ATCOs. We also show that the tool helps ATCOs solving complex problems in a pairwise manner.

\subsection{Background}

Whereas on board systems fully use automation, en-route air traffic controllers who are in contact with pilots still use their mental skills to detect conflicts and order resolution maneuvers. In France, as in many countries with high traffic densities, they have little assistance to accomplish this task. Both conflict detection and resolution require much of ATCO mental resources. The current Air Traffic Management system is organized in filters with a decreasing time horizon. Each filter ensures that the complexity of the next one is tolerable. The airspace is divided in volumes called sectors. The density of traffic inside a sector is regulated in order to avoid too high densities. When the traffic is low, sectors are grouped in order to limit the number of ATCO operating. They are ungrouped when the traffic density increases. However their size cannot be too small in order to keep aircraft maneuverable.

In most countries, ATCO visualize aircraft on a screen using a $2 \mathrm{D}$ horizontal projection. Moving plots represent aircraft and a comet materializes the past positions of the aircraft. A line segment representing the current speed vector and $x$ (3,6 or 9$)$ minutes of flight can be used to project the future positions of the aircraft according to its current speed. ATCOs generally rely on it to detect potential conflicts. On demand, ATCOs can also measure distances between points to check minimum separations.

Many projects have tried to improve the controller's efficiency in the conflict detection task. When building a detection tool the main challenge is to correctly manage uncertainties (e.g., coming from a changing wind or slight deviations of aircraft speed or heading). If a detection tool overestimates uncertainties many detected conflicts will never happen. On the contrary, if it underestimates uncertainties, some conflicts will be missed. In both cases, the ATCO will not trust the tool and abandon its use. Corver and Grote (2016) proposed a field study exploring the sources of uncertainties and the different management strategies adopted by controllers. Building tools to help controllers in their detection and resolution task has been source of experimental research since the early 1990s. In Europe, HIPS [Meckiff and Gibbs (1994); Price and Meckiff (1997)] the Highly Interactive Problem Solver is close to the tool used in our experimentations and was issued from ARC2000 [Dean et al (1995)]. HIPS showed the conflicting zones, called no-go-zones, in an interactive way for one aircraft at a time, as long as the intent of the other aircraft were known. It took into account uncertainties and was tested on diverse scenarios with real ATCOs. [Duong 
et al (1997)] tested HIPS in a Free Flight environment and on Oceanic traffic [Price and Meckiff [1997)]. HIPS did not give any resolution advice but was only meant to dynamically represent the evolution of the no-go-zones. However, HIPS did not give any information on how to return to the original route once the conflict is solved because it did not check conflicts on this leg of the trajectory. Uncertainty was taken into account but the uncertainty model is not well documented in the references. More information was later given by Bakker and Blom (2000) who compare different conflict prediction models taking into account uncertainties: HIPS used geometric approach modeling aircraft by ellipses and measures the distance between ellipses to detect conflicts. The ellipse size grew with time in the speed direction. Bakker and Blom compared the geometric conflict predictor with a probabilistic model used by Erzberger (1997) adopted by Arthur and McLaughlin (1998) in the American project URET (User Request Evaluation Tool). In the US, Erzberger (1997) introduced in the 90s a conflict predictor used by Arthur and McLaughlin (1998). The model could display conflict probabilities in complex situations. Prevot et al (2005) used the conflict probe to assist ATCOs in the conflict detection task. Prevot et al (2008) showed how new displays of conflicts and an interactive conflict solver could help controllers deal with 3 times the current traffic. In Prevot et al (2011) the robustness of the automated solver tool was checked with bad weather conditions and time constraints. Borst et al (2017) introduced a solution space diagram for conflict detection and resolution. It modeled the speed vector and forbidden zones created by other aircraft.

All these experiments showed some possible improvement of the actual system, but they do not isolate the factors that contribute to major improvements.

\subsection{ATCOs' cognitive processes}

Expertise in ATC has been of interest since the 1970s (Bisseret 1971). In such dynamic environments it is thought that ATCOs develop mental models that aid in using strategies and in efficient decision making (Gentner, 2014). ATCO experts seem to follow sufficiently differentiated strategies that allow to solve a wide range of problems (Seamster et al, 1993). Concerning conflict detection, much research was focused on understanding which factors ATCO experts take into account before deciding whether there is a conflict between two aircraft or not (Neal and Kwantes, 2009. Rantanen and Nunes, 2005). For instance, Rantanen and Nunes (2005) found that ATCOs first considered aircraft' altitudes, then extrapolated aircraft trajectories and finally made speed-distance computations. Neal and Kwantes (2009) studied effects of minimum distance at closest point of approach, conflict angle and speed difference on conflict decisions for situations involving two independent conflicts involving two aircraft each.

Cognitive strategies of ATCOs in real work situations were explored by Karikawa et al (2013) and visualization tools of en-route tasks were developed to analyze the performance of controllers' strategies (Karikawa et al, 2014). More recently, Edwards et al (2017) studied the interaction of situation awareness and workload for different levels of automation in a real environment as well. 
Alliot and Bosc (1996) compared the ATCO task to chess playing and chess programs. Among the similarities between chess playing and air traffic control, they pointed out: human individual and intellectual activity, strong time constraint, important theoretical and empirical expertise, short and long term predictions, precisely defined workspace, strong monitoring and psychological constraint. However, they did not focus on the combinatorial aspects of the problems which is another similarity that we try to explain in this paper. Indeed, it is well known that chess experts use pattern recognition rather than testing all the possible moves stemming from one position (Gobet and Charness, 2006). In the same way, when solving a conflict, due to limited cognitive resources, controllers do not evaluate all the possible combinations but rely on pattern recognition of known configurations as well as on general strategies acquired through their experience (Seamster et al, 1993). There are also major differences with chess playing such as predictability, and the consequences of a mistake that can lead to the loss of human lives in air traffic control.

Indeed, we show later in the introduction that for only 3 aircraft, there can be up to 8 different trajectory combinations in the horizontal plane. For 4 aircraft the number of combinations reaches 64 and 1024 for 5 aircraft, which is far more than what a human brain can process, especially in real-time and a stressful environment. Even experienced ATCOs are not supposed to think of so many possible solutions. We introduced a simple dynamic visualization tool that can help simplify complex situations. We call it the Conflict Assistance Tool (CAT). It is meant to help the controller manage complex situations without giving the optimal solution. We deliberately used a simplified environment in order to isolate the intrinsic complexity of conflict resolution in the horizontal plane. We chose to use a single flight level because controllers usually do not combine vertical and horizontal maneuvers and we wanted to explore the resolution options offered by horizontal maneuvers. Our examples were randomly generated clusters of different sizes. We checked how a tool capable of dynamically showing parts of potentially conflicting trajectories could help controllers separate aircraft for different levels of complexity.

Durand et al (2018) showed in previous research using the CAT tool that the initial two year training of trainee ATCOs in France had a low impact on their capacity to manage complex situations. When comparing a group of trainee ATCOs with a group of engineering students who had the same age and scientific background without the ATC training experience, we did not see any significant difference except on delay management. We suspected that trainee ATCOs did not sufficiently practice solving complex situations during their basic training at Aviation school and thus could not optimally solve conflicts involving three to five aircraft, without the CAT tool. However, it would be expected that experienced ATCOs would be able to solve efficiently conflicts involving three or four aircraft, which involve 8 and 64 combinations, respectively, as it corresponds to conflicts that they have to solve in operational settings. Nevertheless, as conflicts involving five aircraft is both very unusual and very complex (1024 combinations), the question remains open whether experienced ATCOs would solve these conflicts efficiently without any visualization tool. One could expect that situations involving five aircraft necessitate the projection of the five future aircraft positions as well as the decisions about which aircraft to deviate and in which direction in order to solve the conflicts. This would be likely 
to load heavily on working memory. Several studies support the view that human working memory is composed at least of a visuospatial component and a central executive component (Baddeley and Logie, 1999). Both components are known to be limited in capacity. Solving an air traffic control situation involves temporary storage of sequences of aircraft locations as well as the processing of this information. As a consequence, as the situation becomes more complex, one can suppose that the working memory capacity limit would impair performance on conflict solving. Moreover, findings in cognitive psychology have highlighted that visuospatial memory and reasoning are intrinsically related (Tabachneck-Schijf et al, 1997). Therefore, the use of an external visualization tool may help ATCOs to complement their internal mental representations and find a solution to the conflict situation (Tversky, 2005).

In this article, we repeated the experiment with experienced ATCOs working in a busy air traffic control center in France (Reims control center is in charge of the north east of France). We do not try to compete with the existing projects to organize the controller's work or assist the conflict detection and resolution task. Our objective is to enlighten the combinatorial underlying difficulty of the tactical horizontal deconfliction task through a simple experiment conducted on trainee and experienced ATCOs with a part-task trainer as defined by Juricic et al (2011).

We particularly focus on the combinatorial aspect of the controller's task. To isolate this difficulty of the resolution task, we conducted a simplified experiment comparing complex conflict situations involving 2, 3, 4 or 5 aircraft with or without the CAT tool. Expertise has been generally shown to be domain-specific (e.g. MylesWorsley et al. 1988). Therefore, experts of one domain are not supposed to perform highly in another domain, unless the required cognitive processes are sufficiently similar. Thus, if experienced ATCOs perform highly on this simplified experiment, we could conclude that cognitive processes to solve conflicts in the simplified environment were somewhat common to those used in real-life.

We did not expect CAT to help experienced ATCOs on easy conflicts but we measured how it helps them in highly complex situations. As we used random examples and a simplified environment, experienced ATCOs were not supposed to use recognition skills that would be specific to the sector they are responsible for in reallife. However, they could use general conflict solving strategies acquired through their years of experience. Moreover, we show that experienced ATCOs perform better than trainee ATCOs without any helping tool on this laboratory task. This means that experienced ATCOs use cognitive skills acquired through their operational experience to solve conflicts even in this simplified ATC environment. This validates the fact that solving conflicts in this simplified environment involves some common cognitive processes with operational air traffic control.

In order to keep this article easy to understand, we decided to include the parts of our initial publication that describe the background, the horizontal conflict resolution complexity, the related bibliography and the experiment description. The new results compare those obtained in the initial experiment (trainee ATCOs) with results performed with experienced ATCOs. 


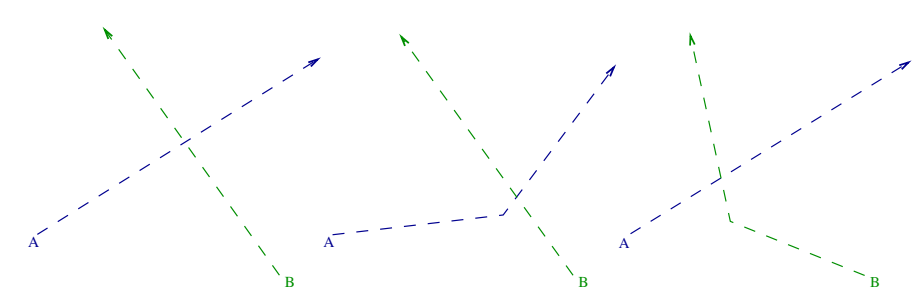

Fig. 1 Two aircraft conflict, the ATCO can decide to turn A to let B go first or turn B to let A go first.

\subsection{Conflict Resolution Complexity}

ATCOs make discrete decisions on aircraft trajectories. They have to decide a specific maneuver at a specific time in an environment that is continuously and slowly changing. However, the problem itself is complex because it is combinatorial. Durand (1996) explained that even if trajectory minimization can be modeled by a convex function, the solution space in the horizontal plane is divided in many unconnected components which oblige controllers to make discrete choices. For example, as shown in Figure 1, the set of conflict free trajectories has two separated components depending on which aircraft passes the trajectories intersection first. Inside each component many trajectory adjustments can be made.

A conflict involving $n$ aircraft has $\frac{n(n-1)}{2}$ different pairs of aircraft. Each pair of aircraft is a potential conflict, and a solution will decide if aircraft in this potential conflict turn right or left of each other if they are facing, or before or after each other in other cases. When combining these possibilities, for $n$ aircraft there may be up to $2^{\frac{n(n-1)}{2}}$ different components in the free trajectory space. For example, if aircraft $A, B$ and $C$ are in conflict, we have 8 conflict resolution options as shown on Figure 2 .

Many of these options are not efficient and do not need to be checked, but they might exist and make the problem very difficult for humans to solve. For 4 aircraft, we have up to 64 options, and for 5 aircraft 1024 options. This strongly suggests that any method that requires exploring every connected component is NP-difficult.

This mathematical complexity is different from the complexity experienced by ATCOs. It is independent from the modeling chosen and even small numbers of aircraft can create huge numbers of options. Durand and Granger (2003) statistically studied the complexity of real traffic data over France. A cluster analysis showed that 2 and 3 aircraft clusters are the majority, but 5 and more aircraft clusters were not rare in the end of the 90s. Because the traffic becomes denser, ATCOs will likely have to deal with the issue of solving more complex clusters.

The cluster structures can vary according to the number of aircraft and conflicts involved. A 5 aircraft cluster can have from 4 to 10 conflicts. If aircraft represent the vertices of a graph and conflicts between them the edges of a graph, a connected graph is a cluster and as shown on Figure 3, there are many different graphs for a same size cluster. For example, for a 5 aircraft cluster, there are 20 different possible structures of clusters, depending on the number or conflicts, and the way they interact. Consequently, one can easily understand how difficult it is for an ATCO to recognize which kind of conflict he/she is facing when the clusters reach such sizes. For a two 

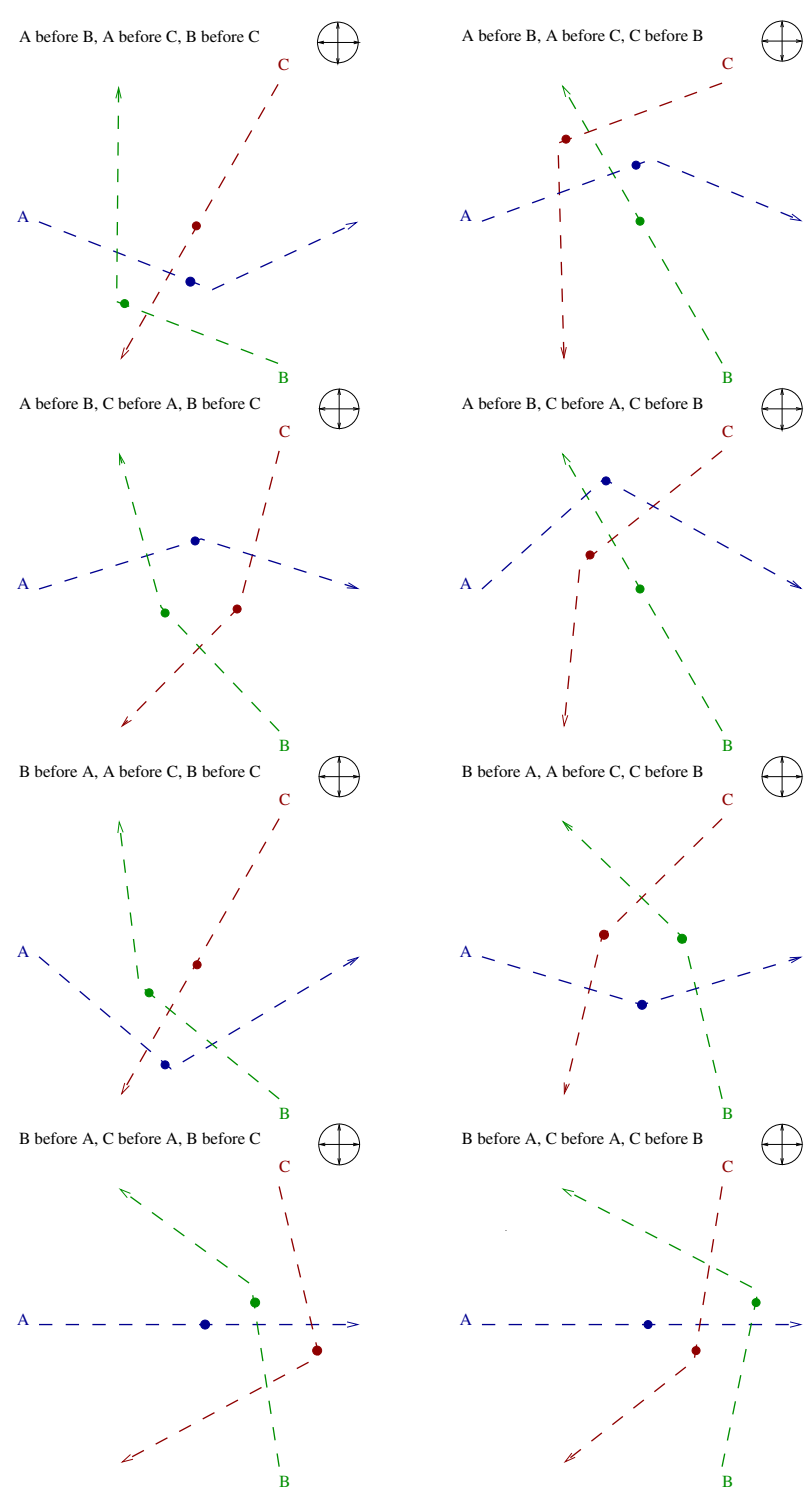

Fig. 28 different conflict resolutions for 3 aircraft. The dot represents the position of aircraft at timestep 7.

aircraft cluster, there is only one possible, structure, for a three aircraft cluster, there are two different structures, one with two conflicts, and one with three conflicts. For a four aircraft cluster, there are six possibilities, two involving three conflicts, two involving 4 conflicts, one involving 5 conflicts and one 6 conflicts. The whole system today is designed to prevent situations involving 5 aircraft that have to be solved horizontally. If the traffic demand continues to grow, these unlikely situations might 

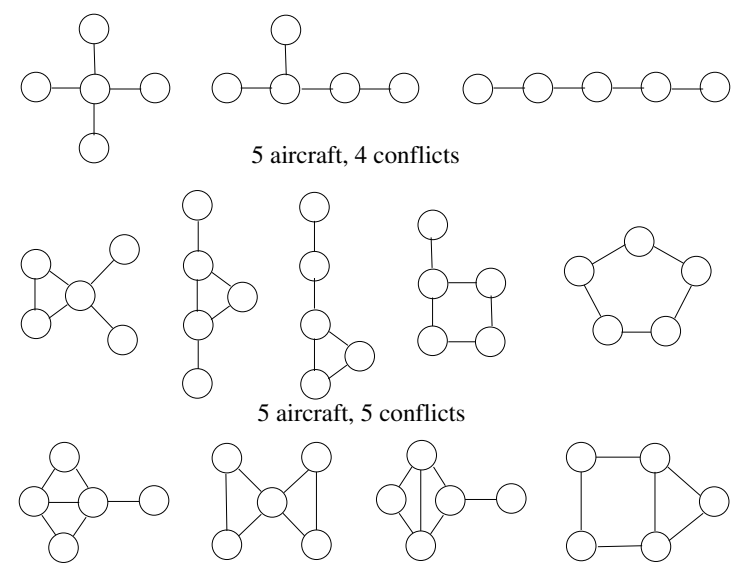

5 aircraft, 6 conflicts
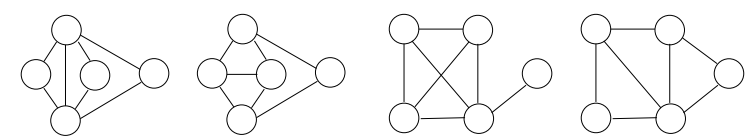

5 aircraft, 7 conflicts
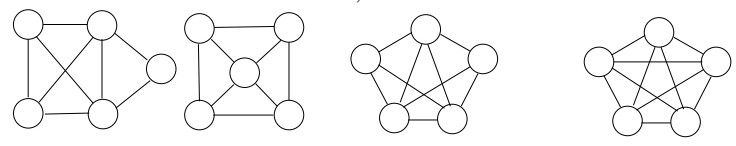

5 aircraft, 8 conflict

5 aircraft, 9 conflicts 5 aircraft, 10 conflicts

Fig. 3 Different structures for 5 aircraft clusters. Each vertex represents an aircraft, each edge is a conflict between two aircraft.

become possible. Our research tries to analyze what can be done horizontally, and why it is difficult for humans to apprehend complex situations horizontally.

\subsection{Outline}

In part2, we describe the tool used in our experiments to test horizontal deconfliction. We detail the two modes of the resolution tool. We explain how it was designed and how it works. We also explain the experiments that took place in Reims with 17 experienced ATCOs. Part 3 analyses the results quantitatively and qualitatively. We conclude and suggest further avenues of inquiry.

\section{Experiment Description}

\subsection{Tool description}

We deliberately isolated the horizontal deconfliction task in order to show the limits of humans with the existing tools on this specific task. We built a part-task trainer, using a 2D-display of random traffic, with no pre-defined routes. This display is 


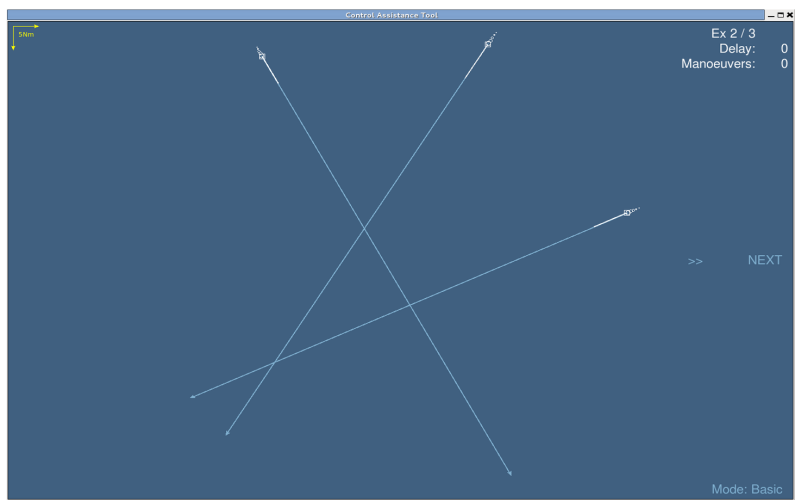

Fig. 43 aircraft conflict in the Basic mode.

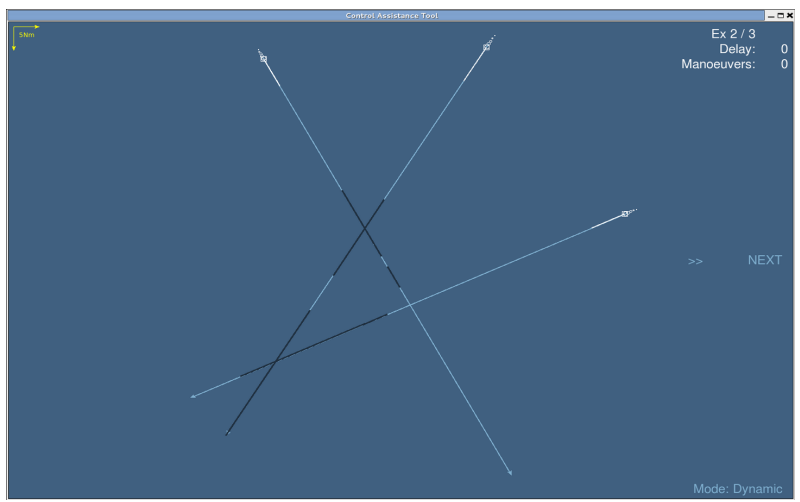

Fig. 53 aircraft conflict in the Dynamic mode.

equipped with a simple tool using the computer mouse to change aircraft trajectories. The tool is rather simple and intuitive. Aircraft are represented by plots. A comet shows the past position and the speed vector is represented by a segment showing the future position of the aircraft in one minute. The five nautical miles separation standard is displayed on the top left of the screen.

The trajectory track is represented as a light line on which the plot representing the aircraft moves. In the experiments, we compare two modes of conflict display. In the Basic mode, no information of the conflict location is given on the trajectory track. Figure 4 gives an example of a 3 aircraft conflict in the Basic display mode. In the Dynamic display mode, the part of the trajectory of each aircraft in conflict is represented by black segments. Figure 5 shows the same 3 aircraft example in the Dynamic mode.

For the experiments, the double white arrow allows the user to move forward in time and the NEXT button moves to the next exercise. In both modes, the user can modify the trajectory with the cursor. When the user positions the cursor over a trajectory line, it turns blue. The user can select a point of the trajectory by holding the left click. While holding the left click the user can move the trajectory line and add 


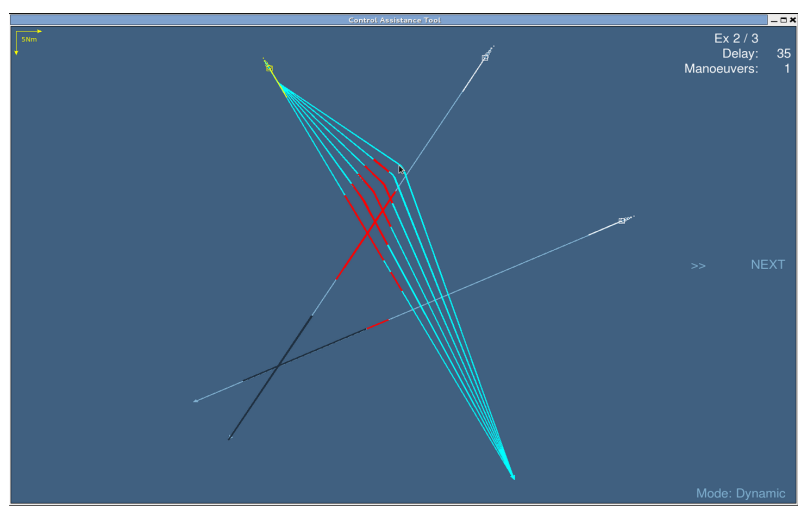

Fig. 6 Mouse interactions in the Dynamic mode.

a maneuver when releasing it. A right click during the move cancels the maneuver. The number of maneuver actions and the delay caused by maneuvers are displayed on the top right of the screen.

In the Dynamic mode, conflicting parts of trajectories are represented in black. Because of uncertainties, these parts can shrink or vanish with time. When the cursor selects a trajectory (blue), the conflicting parts involving this trajectory turn red. When selecting a point of the trajectory and moving it, the user can dynamically see the evolution of the conflict zone (see Figure 6.

The experiment starts with a short text explaining the display and the mouse functions. It explains that aircraft are represented by plots, comets show the past positions and the speed vectors give the expected positions within one minute. It reminds that the separation standard is on the top left of the screen. The mouse functions are detailed as follows:

- By clicking and holding the left button, the user can catch and move a point of the trajectory and create a maneuver when releasing the button;

- While moving a point, a right click cancels the move;

- Moving the mouse wheel advances the time;

- The Next button moves to the next exercise.

Before the set of Basic mode exercises we give one page of guidelines to inform the participants that conflict zones are not represented. We remind the priorities: they must first solve conflicts, second minimize the number of maneuvers, and third limit the delay. The first exercise is a training exercise.

Before the set of Dynamic mode exercises we give one page of guidelines to inform the participants that conflicting parts of trajectories are represented in black. We inform them that these parts can shrink or vanish with time because of uncertainty. When a trajectory is selected, it turns blue and the conflicting parts involving this trajectory turn red. We also remind the priorities: they must first solve conflicts, second minimize the number of maneuvers, and third limit the delay. The first exercise is a training exercise. 


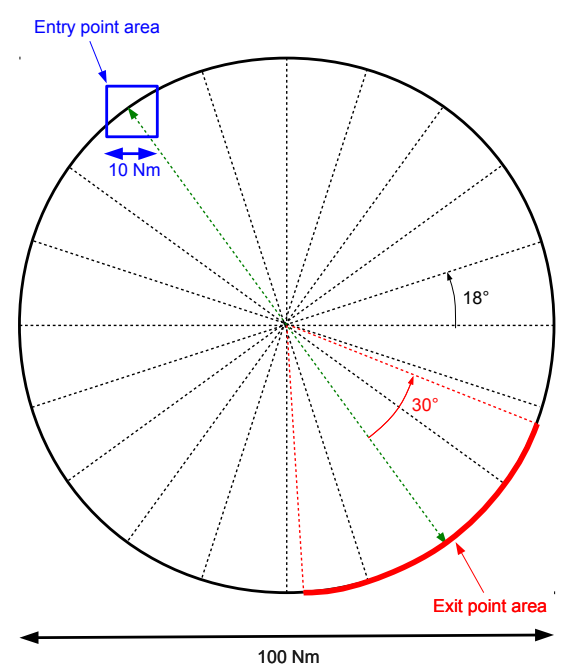

Fig. 7 Generation of random traffic situations.

\subsection{Conflict Detection Calculation}

The conflict detection calculation was detailed by Durand et al (2018). Each trajectory is divided in line segments on which the aircraft has a constant speed. We use a standard rate ( 3 degrees per second) to model turns and approximate turns with segments. The angle between two consecutive segments cannot exceed 10 degrees in order to keep the trajectory display smooth. To measure the separation between two trajectories, we add points on both trajectories in order to synchronize the lists of segments. Once segments are synchronized, the problem is reduced to checking the distance between segments. We add some uncertainty in the model by increasing the separation standard linearly with time. Using such a growing norm is very convenient for quickly calculating the conflicting zone, but it can only model an isotropic growth of uncertainty. This is the main problem of such a model.

In real life, controllers often wait until the conflict is certain before maneuvering an aircraft. This simple isotropic model is not completely realistic but it is able to model the interest of waiting before acting by showing conflicting zones that can either shrink with time if the conflict disappears or remain if not. This aspect is essential to build experiments where predicted conflicts integrate uncertainties on the trajectory prediction.

\subsection{Exercise generation}

In order to generate some random traffic situations with different types of conflicts between aircraft, we consider a circular sector with a diameter of 100 nautical miles (about 15 minutes of flying time for an aircraft), with 20 possible entry points regu- 

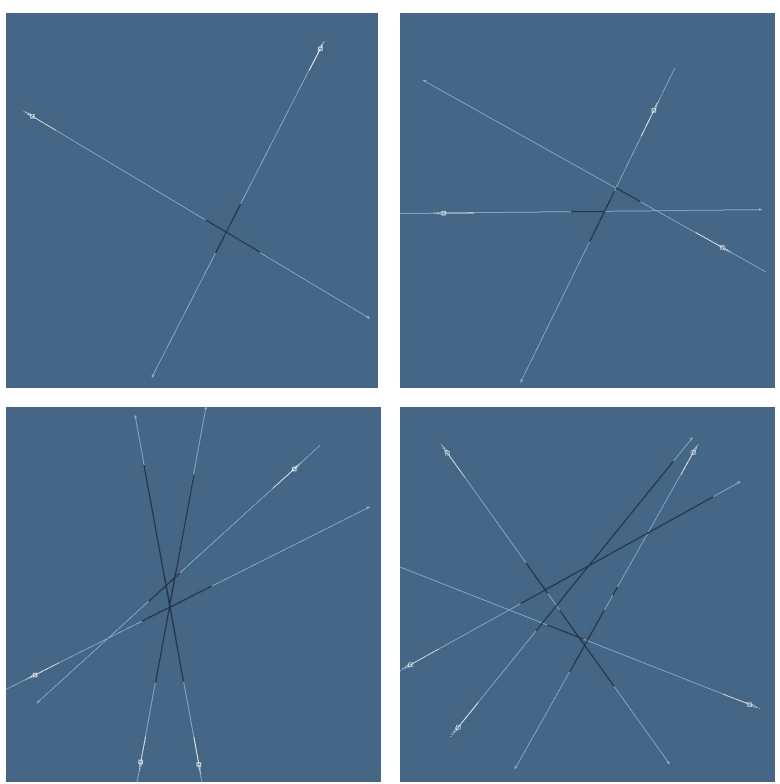

Fig. 8 Examples of traffic situations, with 2, 3, 4 and 5 aircraft.

larly positioned on its circumference (see Figure 7). With these orders of magnitude, the distance between two neighboring entry points is over 15 nautical miles (which is three times greater than the minimal separation distance between aircraft).

The number of aircraft in the traffic situations vary from 2 to 5 . Each aircraft is randomly assigned:

- a nominal speed, between 370 and 550 knots;

- its own entry point, in a rectangular area of 10 nautical miles around one of the sector's entry points;

- an exit point on the opposite side of the sector, in a slice extending by plus or minus 30 degrees around the opposite point on the circle.

Initially, each aircraft flies directly from its entry point to its exit point.

In order to avoid unmanageable traffic situations, the following constraints were required additionally (situations not respecting these constraints were discarded):

- A minimal duration of 3 minutes was required before the first conflict happens.

- A conflict solver using a genetic algorithm, as described by Durand et al (1996), was run to check that a solution exists using some simple maneuvers defined by three values per aircraft $\left(t_{0}, t_{1}, \alpha\right)$ : the aircraft turns $\alpha$ degrees at $t_{0}$ and resumes its course at $t_{1}$, with the following ranges:

- $\alpha \in\left[-40^{\circ}, 40^{\circ}\right]$

- $t_{0} \in[0,10]$ (minutes)

- $t_{1} \in\left[t_{0}, 10\right]$ (minutes)

Figure 8 gives some examples of such traffic situations, displayed in the Dynamic mode. Using this process, two series of 16 exercises were generated for the exper- 
iment: each series is intended to be run in a different mode (Basic or Dynamic) by each group of participants, and contains in the following order:

\begin{tabular}{|ll|}
\hline 1 training situation & with 2 aircraft \\
\hline 3 situations & with 2 aircraft \\
\hline 4 situations & with 3 aircraft \\
\hline 4 situations & with 4 aircraft \\
\hline 4 situations & with 5 aircraft \\
\hline
\end{tabular}

In sum, each participant realized 30 exercises in total (and one training situation for each mode).

\subsection{Participants}

We conducted the experiment with two categories of Air Traffic Controllers:

- 20 Trainee Air Traffic Controllers (ATCOs), that were at the end their training at ENAC school in Toulouse;

- 17 Experienced Air Traffic Controllers (ATCOs) from Reims En-Route Control Center.

All trainee ATCOs were between 21 and 24 year old. Experienced ATC0s were between 26 and 50 year old with a 32,7 year old mean age. There were 14 males and 6 females in the trainee group and 12 males and 5 females in the experienced group.

Each category was divided into two groups, which ran the experiment at the same time in the same computer room:

- The first group did the first series of exercises in the Basic mode and the second series in the Dynamic mode

- The second group did the first series of exercises in the Dynamic mode and the second series in the Basic mode

Exercises were randomized for the same number of aircraft but we kept an increasing number of aircraft. There may be a learning effect over time, but it would affect both series of exercises and does not prevent us from concluding on complexity issues.

There was no time constraint. The whole exercise lasted from 30 to 45 minutes. Before the experiment, each participant was asked to read and sign an agreement form, allowing us to use the results collected anonymously.

\subsection{Measures}

During the experiment, each participant tried to solve the different traffic situations one by one, by modifying some aircraft trajectories. For each exercise, the following information was recorded:

- All the mouse actions and the resulting aircraft trajectories, with two time indications: 
- the relative time at which it happened in real life (counted from the beginning of the exercise);

- and the corresponding relative time in the simulation (which is often different as the participant moves forward in time during the exercise).

- The number of aircraft pairs for which some conflicts were not solved at the end of the exercise (i.e. for which the minimal separation distance of 5 nautical miles was not ensured during some periods).

- The number of modified aircraft trajectories.

- The cumulative delay (in seconds) that were generated by the different maneuvers.

- The time spent handling each exercise (in real life).

The collected data are accessible at:

https://cloud.recherche.enac.fr/index.php/s/MqAQsQBaJ8a72ax

\section{Results}

\subsection{Presentation of results}

In the following sections, we analyzed three performance parameters corresponding to the three objectives given to the participants:

1. The number of unsolved conflicts;

2. The number of maneuvers used to solve conflicts;

3. The delays of maneuvered aircraft.

The participants had to minimize each of these three parameters, in this order of priority. Moreover, two parameters gave indications about how participants processed the information:

4. The number of mouse actions;

5. The time spent on the exercises.

The results were gathered by number of aircraft and by experiment mode (Basic or Dynamic): each value is the average across participants and across exercises, for a given number of aircraft and a given experiment mode. Except for the number of unsolved conflicts, analyzes of the other parameters included only solved exercises. The proportion of solved exercises represented 52.5 to $93.3 \%$ for trainee ATCOs and 76.4 to $98.0 \%$ for experienced ATCOs depending on the number of aircraft involved.

For each of the five parameters, we assessed the potential effects of the group of participants, the difficulty of the exercise, i.e., the number of aircraft, the experiment mode and their interaction. The residuals of a linear model of these factors were not normally distributed for any of the five parameters ( $p<.001$ for all Shapiro tests). Therefore, for each of the five parameters, we could not analyze the data with an ANOVA (analysis of variance) and fitted the data to a generalized least square (GLS) model, using the rms package of R (version 3.1.1) [R Core Team (2018)]. This GLS model analyzed the effect of following variables and their interactions: Group (trainee ATCOs vs. experienced ATCOs), Number of aircraft (2, 3, 4 or 5) and Mode (Basic vs. Dynamic). 


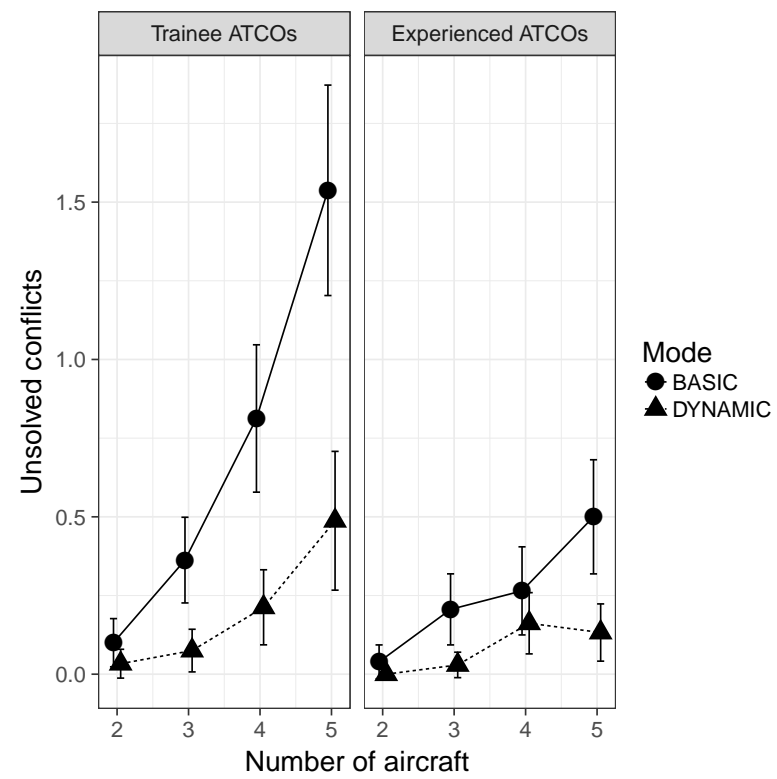

Fig. 9 Mean number of unsolved conflicts for each mode (Basic or Dynamic), group (trainee ATCOs or experienced ATCOs) and number of aircraft (2 to 5). Error bars represent 95 confidence intervals.

\subsection{Unsolved conflicts}

The first goal assigned in the instructions was to solve the conflicts: Figure 9 shows the number of unsolved conflicts, measured by the number of aircraft pairs that still have a conflict in the proposed solution.

After fitting a GLS model, we can state that there was a main effect of the Group $(t=3.68, p<.001)$ on the number of unsolved conflicts and of the Mode $(t=3.37$, $p<.001)$. Moreover, the interaction Group x Mode $\mathrm{x}$ Number of aircraft $(t=3.11$, $p .002$ ) significantly contributed to explaining the variance of the number of unsolved conflicts (see Table I for descriptive statistics). More precisely, if we consider experienced/trainee differences depending on the Mode and the number of aircraft:

- with two aircraft there was no significant difference between trainee ATCOs and experienced ATCOs in any Mode (Basic or Dynamic).

- in the Basic Mode, from three to five aircraft, trainee ATCOs solved significantly less conflicts than experienced ATCOs. For example, with three aircraft, estimated $95 \%$ confidence intervals of the number of unsolved conflicts are $[0.36 ; 0.54]$ for trainee ATCOs and $[0.08 ; 0.28]$ for experienced ATCOs.

- in the Dynamic Mode, there was no significant difference between trainee ATCOs and experienced ATCOs in the number of unsolved conflicts from two to four aircraft, and only a marginally significant difference in cases involving five aircraft. Indeed, estimated $95 \%$ confidence intervals of the number of unsolved conflicts are $[0.30 ; 0.56]$ for trainee ATCOs and $[0.02 ; 0.30]$ for experienced ATCOs. 
In addition, when we focus on differences between the Basic and Dynamic Mode depending on the Group and the number of aircraft:

- Trainee ATCOs solved significantly more conflicts in the Dynamic Mode than in the Basic Mode from three to five aircraft.

- Experienced ATCOs solved significantly more conflicts in the Dynamic Mode than in the Basic Mode only for five aircraft: estimated 95\% confidence intervals of the number of unsolved conflicts are [0.32;0.61] for the Basic Mode and $[0.02 ; 0.30]$ for the Dynamic Mode. Experienced ATCOs solved $88.2 \%$ of the 5 aircraft conflicts in the Dynamic Mode and only $64.7 \%$ of them in the Basic Mode.

These results provide the main contribution of this experiment: except for the simplest cases (two aircraft), more conflicts were solved with the Dynamic Mode for trainee ATCOs. The experienced ATCOs started taking advantage of the Dynamic mode in the most complex cases (five aircraft) and not in the easier ones (from two to four aircraft).

These results confirm that making the conflicting portions of trajectories dynamically visible while modifying one of them provides significant assistance in solving the conflicts, even though it has to be done step by step. These results also confirm the difficulty for humans to find solutions for conflicts involving many aircraft, even on problems for which the genetic algorithm solver finds instantly a simple solution involving no more than one maneuver per aircraft.

\begin{tabular}{|c|c|c|c|c|c|c|c|c|c|c|c|c|}
\hline & & & \multicolumn{2}{|c|}{ Unsolved confs } & \multicolumn{2}{|c|}{ Maneuvers } & \multicolumn{2}{|c|}{ Delay (s) } & \multicolumn{2}{|c|}{ Actions } & \multicolumn{2}{|c|}{ Time (s) } \\
\hline Mode & Group & Acft & $M$ & $S D$ & $M$ & $S D$ & $M$ & $S D$ & $M$ & $S D$ & $M$ & $S D$ \\
\hline Basic & Trainee ATCOs & 2 & 0,10 & 0,30 & 1,25 & 0,57 & 33,35 & 29,63 & 1,85 & 1,36 & 24,03 & 11,12 \\
\hline Dynamic & Trainee ATCOs & 2 & 0,03 & 0,18 & 0,95 & 0,77 & 24,33 & 31,55 & 1,63 & 2,05 & 22,48 & 13,00 \\
\hline Basic & Exp. ATCOs & 2 & 0,04 & 0,20 & 1,25 & 0,66 & 33,22 & 39,35 & 1,82 & 1,32 & 18,98 & 14,14 \\
\hline Dynamic & Exp. ATCOs & 2 & 0,00 & 0,00 & 1,00 & 0,82 & 18,61 & 19,11 & 1,43 & 1,51 & 21,12 & 20,04 \\
\hline Basic & Trainee ATCOs & 3 & 0,36 & 0,62 & 2,08 & 0,78 & 87,08 & 99,00 & 3,08 & 2,02 & 37,90 & 23,85 \\
\hline Dynamic & Trainee ATCOs & 3 & 0,08 & 0,31 & 2,28 & 0,69 & 74,59 & 66,90 & 4,46 & 3,53 & 49,15 & 24,59 \\
\hline Basic & Exp. ATCOs & 3 & 0,21 & 0,48 & 2,16 & 0,68 & 97,06 & 74,03 & 3,16 & 1,62 & 33,28 & 18,18 \\
\hline Dynamic & Exp. ATCOs & 3 & 0,03 & 0,17 & 2,32 & 0,66 & 90,09 & 70,00 & 3,60 & 2,34 & 36,84 & 32,12 \\
\hline Basic & Trainee ATCOs & 4 & 0,81 & 1,07 & 2,85 & 1,06 & 129,68 & 108,58 & 4,39 & 2,43 & 49,74 & 23,18 \\
\hline Dynamic & Trainee ATCOs & 4 & 0,21 & 0,54 & 2,76 & 1,21 & 98,89 & 100,14 & 5,79 & 5,19 & 60,69 & 42,06 \\
\hline Basic & Exp. ATCOs & 4 & 0,26 & 0,59 & 2,75 & 1,01 & 137,56 & 109,71 & 4,54 & 2,83 & 48,74 & 34,70 \\
\hline Dynamic & Exp. ATCOs & 4 & 0,16 & 0,41 & 3,07 & 0,98 & 148,21 & 136,56 & 5,84 & 4,57 & 54,81 & 37,72 \\
\hline Basic & Trainee ATCOs & 5 & 1,54 & 1,53 & 3,58 & 0,92 & 193,50 & 113,05 & 5,86 & 2,32 & 57,04 & 27,09 \\
\hline Dynamic & Trainee ATCOs & 5 & 0,49 & 1,01 & 3,36 & 1,23 & 141,14 & 103,39 & 7,69 & 6,55 & 75,58 & 52,58 \\
\hline Basic & Exp. ATCOs & 5 & 0,50 & 0,76 & 3,79 & 1,10 & 216,66 & 139,58 & 6,44 & 2,99 & 68,00 & 41,93 \\
\hline Dynamic & Exp. ATCOs & 5 & 0,13 & 0,38 & 4,06 & 0,75 & 250,79 & 189,34 & 8,47 & 4,03 & 85,78 & 53,13 \\
\hline
\end{tabular}

Table 1 Means and standard deviations of the dependent variables according to the group (trainee ATCOs vs. experienced ATCOs), mode (Basic vs. Dynamic) and number of aircraft (from 2 to 5). 


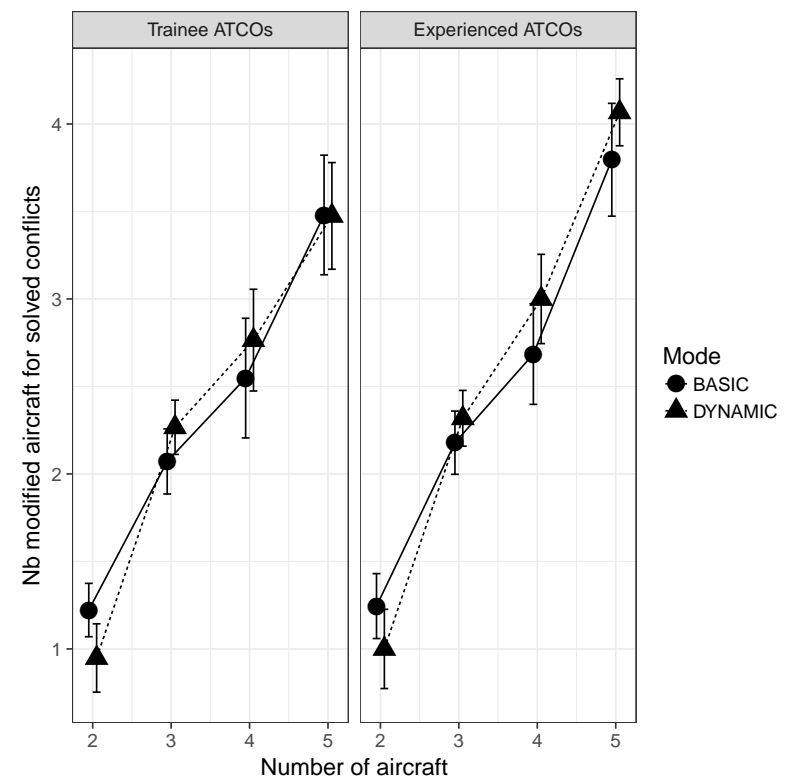

Fig. 10 Average number of modified aircraft trajectories for each mode (Basic or Dynamic), group (trainee ATCOs or experienced ATCOs) and number of aircraft (2 to 5), restricted to solved conflicts only. Error bars represent 95 confidence intervals.

\subsection{Number of maneuvers}

The guidelines of the experiment also asked participants to minimize the number of maneuvers (after solving the conflicts).

In the recorded data, one way to assess the number of maneuvers is to count the number of aircraft trajectories that were modified, while ignoring the number of mouse interactions that were actually needed, as shown in Figure 10

From the GLS model, we can state that the number of modified aircraft trajectories increases with the number of aircraft involved in the case $(t=10.93, p<.001)$. Neither the Group $(t=0.61, p=.54)$, nor the Mode $(t=0.82, p=.41)$ contributed significantly to explain the variance of the number of modified aircraft. About $n-1$ aircraft trajectories are modified on average for a case involving $n$ aircraft. Indeed, in the Basic mode for trainee ATCOs the estimated 95 confidence intervals were [1.1;1.5], [1.8;2.1], [2.5;2.9] and [3.1;3.7] for cases involving two, three, four and five aircraft, respectively. Confidence intervals for the Dynamic mode and for experienced ATCOs, were non significantly different from these. This can be explained by the fact that participants solve complex conflicts by dealing with one aircraft pair at a time. For each conflicting aircraft pair, participants typically moved only one of the two aircraft in order to avoid the other (and if possible other already maneuvered aircraft). At the end of this process, it looks like participants sorted conflicts by priority, and in most cases, they left one aircraft trajectory completely unchanged (as if this aircraft had the highest priority). 


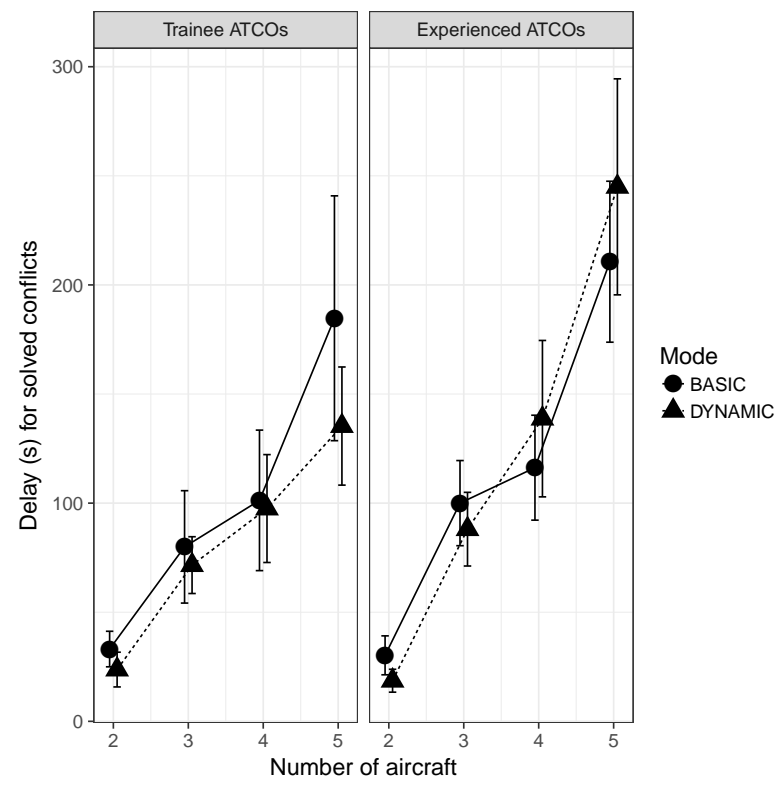

Fig. 11 Average delay (seconds). Error bars represent 95 confidence intervals.

\subsection{Delay}

The third guideline given to the participants was to limit the delay (after solving the conflicts and minimizing the number of maneuvers). Figure 11 shows how the total delay per exercise increases with the number of aircraft, in the two modes.

From the GLS model we can state that only two variables contributed significantly to explaining the variance of the delay: Number of aircraft $(t=6.2, p<.001)$ and the interaction Group x Mode x Number of aircraft $(t=2.2, p=0.03)$. Indeed, the delay globally increases with the complexity of the exercises with no significant difference between both modes from two to four aircraft for trainee ATCOs and experienced ATCOs.

Comparing the delay requires to work on the exact same exercises, and to consider only solved conflicts: By not solving conflicts you may obtain a smaller delay, and depending on the exercise the necessary delay can be different.

Comparing the delay between the the two groups for 5 aircraft conflicts does not make sense because the experienced ATCOs solved more of them, and there is a chance that the conflicts solved by both groups and modes were the "easiest ones" requiring less delay. Nevertheless, there is no significant difference in the delays obtained with five aircraft between the Basic and Dynamic mode for each group separately. 


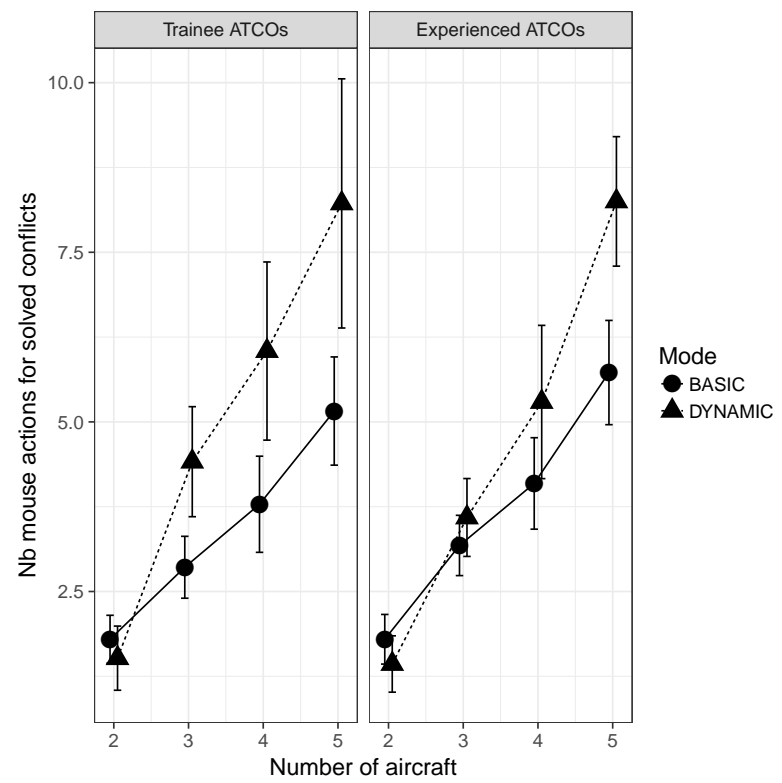

Fig. 12 Average number of mouse actions for each mode (Basic or Dynamic), group (trainee ATCOs or experienced ATCOs) and number of aircraft ( 2 to 5), restricted to solved conflicts only. Error bars represent 95 confidence intervals.

\subsection{Number of mouse actions}

The number of mouse actions needed to handle the exercise gives an estimation of the number of maneuver adjustments. This value overestimates the effective number of maneuvers because a single and simple maneuver can be adjusted several times with different consecutive mouse actions. Furthermore, this value also provides interesting feedback on the complexity of the maneuver elaboration. We can observe in Figure 12 that users tend to interact more with the mouse in the Dynamic mode.

When analyzing results of GLS modeling on this parameter, only two variables contributed significantly to explaining the variance: Number of aircraft $(t=4.3, p<$ $.001)$ and the interaction Mode $\mathrm{x}$ Number of aircraft $(t=3.4, p<.001)$. We can state that:

- In cases involving only two aircraft, there is no significant difference between the number of mouse actions observed with both modes ( 95 confidence intervals were [1.0;2.5] for the Basic mode and [1.1;2.5] for the Dynamic mode for trainee ATCOs, and similar confidence intervals for experienced ATCOs).

- In cases involving five aircraft, the number of mouse actions is significantly higher with the Dynamic mode than with the Basic mode both for trainee ATCOs and experienced ATCOs: [7.6;9.0] for the Dynamic mode and [4.0;6.0] for the Basic mode for the trainee ATCO group and [7.2;8.7] for the Dynamic mode and $[4.8 ; 6.4]$ for the Basic mode for experienced ATCOs. 


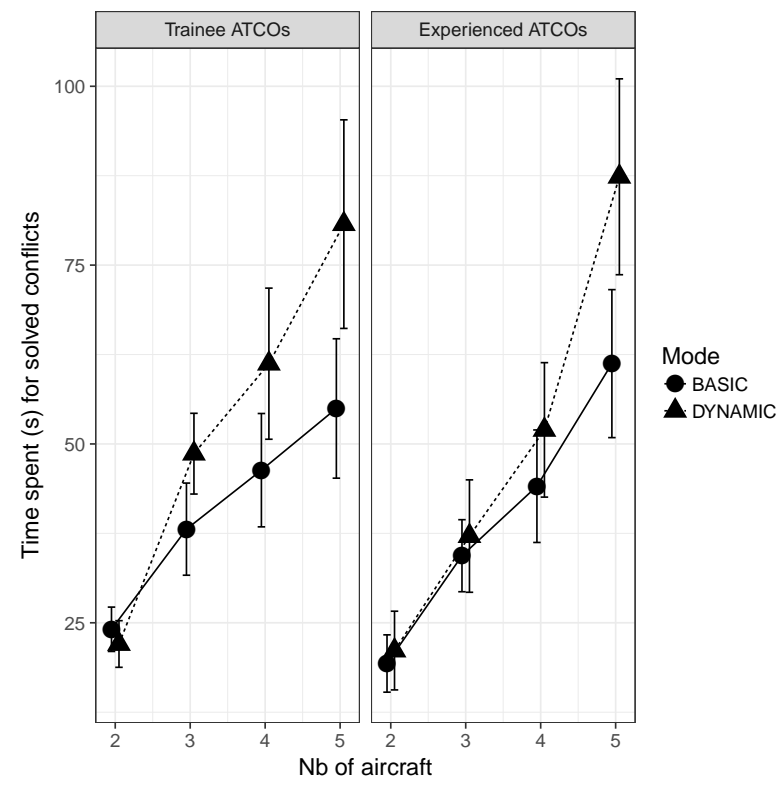

Fig. 13 Average time spent to handle the exercises (seconds) for each mode (Basic or Dynamic), group (trainee ATCOs or experienced ATCOs) and number of aircraft (2 to 5), restricted to solved conflicts only. Error bars represent 95 confidence intervals.

This result is at first surprising because the highlighting of the conflicting portions of the trajectories should theoretically help to define more efficiently all the needed maneuvers, resulting in fewer mouse actions. In fact, it appears that the participants were neither prompted nor trained to take advantage of this mode for this purpose (minimizing the number of mouse actions). On the contrary, the Dynamic mode encouraged them to test different options of maneuvers, in a "what if" way of thinking.

Because the Dynamic mode gives instant feedback on the user's action, the user may be more tempted to adjust the previous actions to reduce delays.

Future experiments with different guidelines and with participants trained in the Dynamic mode could help us confirm this first analysis.

\subsection{Time spent handling the exercises}

Similarly to trends for mouse actions, Figure 13 shows that time spent to handle the exercises increased with the number of aircraft, for both trainee ATCOs and experienced ATCOs.

When analyzing results of GLS modeling on this parameter, only two variables contributed significantly to explaining the variance: Number of aircraft $(t=4.3, p<$ $.001)$ and the interaction Mode $\mathrm{x}$ Number of aircraft $(t=2.7, p=.006)$. We can state that: 
- In cases involving only two aircraft, there is no significant difference between the time spent to handle the exercises in both modes (95 confidence intervals were [18.1;33.1] for the Basic mode and [18.5;32.2] for the Dynamic mode for trainee ATCOs, and similar confidence intervals for experienced ATCOs.

- In cases involving five aircraft, the time spent is significantly longer with the Dynamic mode than with the Basic mode both for trainee ATCOs and experienced ATCOs: [74.6;88.4] for the Dynamic mode and [46.9;66.3] for the Basic mode for trainee ATCOs and [74.6;88.7] for the Dynamic mode and [52.0;67.8] for the Basic mode for experienced ATCOs.

During the experiment, the participants did not have any time limit and had no reason to rapidly solve the exercises. They therefore took more time to explore the different solutions for each aircraft in the Dynamic mode, because they had more information about the transformations of the conflict areas.

\subsection{Qualitative results}

The experienced ATCOs were asked what they thought about the tool at the end of the exercises. Among the positive remarks, we noticed:

- Some experienced ATCOs found that the tool was useful to analyze conflicts and decide which aircraft to move in the right direction.

- They found useful to be able to visualize the second leg of the trajectory, mainly because it guarantees that the resolution is completed.

- The tool allows to check the efficiency of the maneuver and makes sure it guarantees no new conflict.

Among negative remarks:

- Some experienced ATCOs found the tool not easy to use, and not intuitive.

- Some experienced ATCOs regretted the fact that the tool did not actually globally solve the conflict, but only give pairwise indications of the conflicting zones.

- Some experienced ATCOs regretted that the experiments were not realistic and did not include their usual environment. They felt like their usual working methods were not applicable.

These negative remarks were expected because we deliberately chose to isolate the horizontal combinatorial factor conflict resolution.

Among the general remarks concerning the strategies used, experienced ATCOs reported that they used the same general strategies with and without the tool. More specifically they cited two strategies : (i) the strategy of the roundabout and (ii) the strategy of the delay triangle. The first strategy was specifically used when a large number of aircraft were in conflict and consisted in choosing a direction of rotation and rolling up aircraft one behind the other. The second strategy consisted in giving a large heading to the second aircraft at the crossing point to wrap it behind the first one.

In order to better understand the cognitive strategies that experienced ATCOs used with and without the CAT tool, we compared the best solutions found by them in both 
cases. The term best refers to the solution that maximizes the criteria as they were given in the instructions. Thus, the best solution is the solution, among those found by expert controllers, that (i) solves all the conflicts, (ii) used the minimum number of maneuvers and (iii) minimized the delays. For the most complex situations (involving five aircraft) the best solution found was mostly of better quality with the tool than without. What characterized the solution found with the tool was a more optimized solution regarding the number of maneuvers and the delays with less useless margins than without the tool (see Figure 14p. This tendency to use conservative strategies without the tool is consistent with

Bisseret (1981)'s findings. Indeed, in his experiment expert controllers made more conservative conflict judgments than novices. This is also consistent with interviews with controllers which underlined that selecting appropriate control strategies of air traffic, was a key feature of ATCO's skills allowing to handle traffic by reserving their cognitive resources (Karikawa et al, 2014). One can imagine that the tool helped choosing solutions that would require more cognitive resources to monitor the evolving situation in order to ensure safety.

\section{Conclusion and Further Work}

To conclude, we first confirmed with our experiments that the horizontal separation task is difficult for both trainee and experienced ATCOs even if the experienced ATCOs can handle more complex situations. Isolating this task helps to understand that it can be the bottleneck of traffic density increase when the different flight levels are fully used. We also showed that the Dynamic mode helped trainee ATCOs in all situations except the simplest ones (involving only 2 aircraft) and experienced ATCOs for the most complex situations (involving 5 aircraft). In the Basic mode, both trainee and experienced ATCOs started having major issues when clusters involved 5 aircraft. The lack of vertical maneuvers made the exercises more complicated for those cases, but it experimentally confirmed that horizontal separation is a combinatorial problem. In the Dynamic mode, experiments showed that the proposed tool helps the users deal with cluster complexity even if conflicts are generally solved one after another in an iterative manner. There was no significant reduction of the number of maneuvers or delay with the Dynamic mode. It seems that the tool does not provide any clue to the user about how to reduce the number of actions. The reactive aspect of the tool led to more mouse manipulations. By analyzing one by one the answers, we found that the user tended to use the Dynamic mode like a "what if" mode to check options. This can also explain why the time spent solving the exercises was longer with the Dynamic mode.

Experienced ATCOs gave mixed feedback on the experiments. Some were very interested and wished that the tool would even go further, others were disturbed by the lack of realism of the tool. We think that it could be useful to adapt the experiment to a typical Reims scenario in order to offer trainee ATCOs a simplified training tool on which they could learn how to deal with simple and more complex situations, before having to deal with the whole ATC environment. We started to build the tool but noticed some issues that need to be fixed: 
Basic mode - Best solution

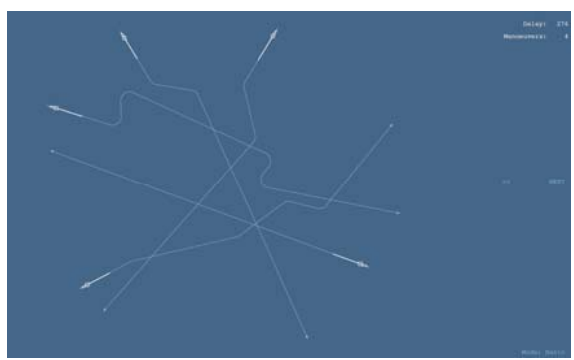

Sit 2

Sit 3
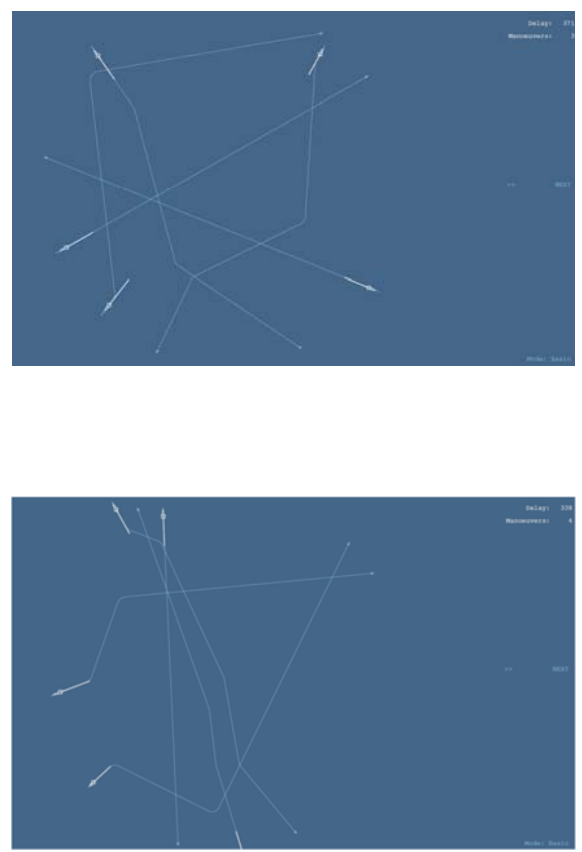

Sit 4

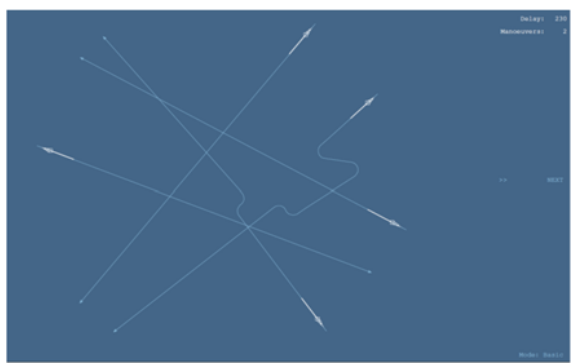

\section{Dynamic mode - Best solution}
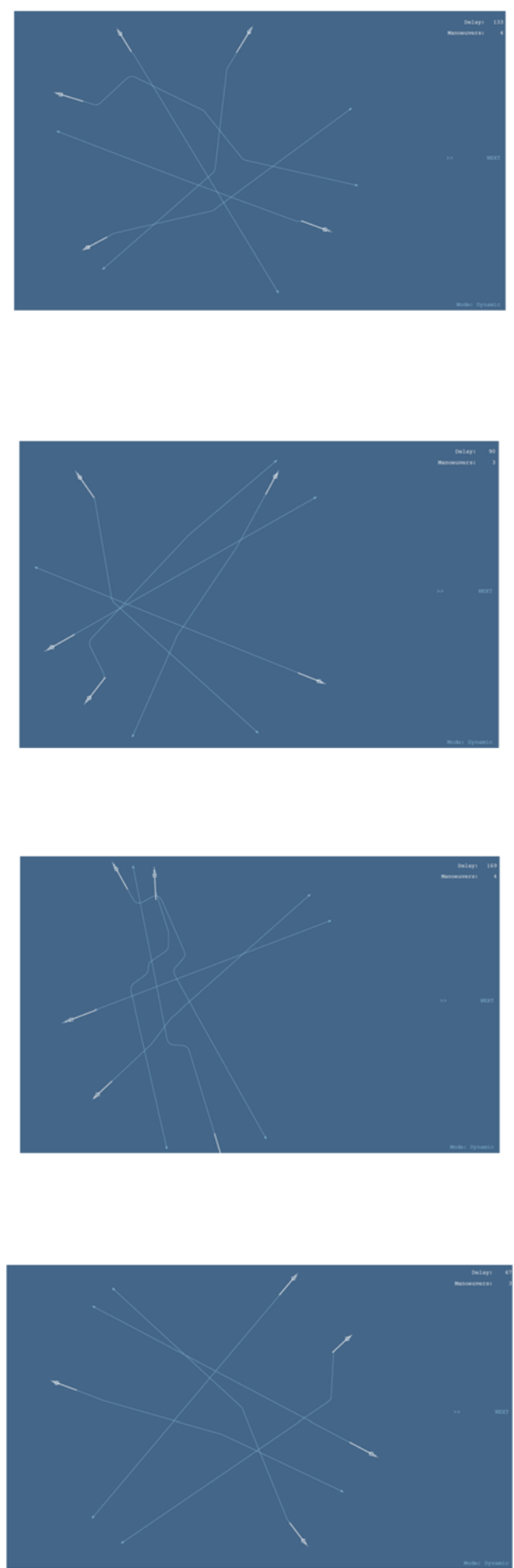

Fig. 14 Examples of best solutions found for five aircraft situations by experienced ATCOs. The term best refers to a solution that maximizes the criteria as they were given in the instructions. Thus, the best solution is the solution, among those found by expert controllers that (i) solves all the conflicts, (ii) used the minimum number of maneuvers and (iii) minimized the delays. On the left panel, the best solution found without the CAT tool and on the right panel the best solution found with the CAT tool. 
- We need to be able to select a trajectory easily when aircraft fly on the same track. This could probably be fixed by adding an aircraft selection mode before changing the trajectory.

- We also need to add vertical maneuvers and find an appropriate way to select vertical maneuvers. This will allow us to understand if the horizontal complexity is reduced or increased when playing with different levels.

- We also need to improve the uncertainty model. First we want to make it compliant to realistic uncertainties. In addition to uncertainties due to wind and speed prediction in both vertical and horizontal planes, we need to model the uncertainty caused by the pilot answer to orders and the track accuracy, especially when aircraft are following headings. We can also imagine that the controller could adjust in real time the uncertainty parameters in order to comply to his/her own preferences.

- We finally project to use real traffic to build scenarios and to be able to compare the efficiency of the tools with real data.

Other ATC decision aids have been evaluated with the more direct objective to be implemented in operational settings. For instance, Trapsilawati et al (2016) assessed a conflict detection and resolution advisory tool, the CRA. This tool shows predicted conflicting pairs of aircraft, the resolution maneuver advisory is explicitly written (e.g., "aircraft A climb FL140") and the controller can accept or reject the proposed resolution. The philosophy of our $C A T$ tool was on the contrary to let the controller decide which maneuvers to implement. Trapsilawati et al (2016) showed higher performance, lower workload and no decrease in situation awareness with the CRA tool. However, the present study illustrated that for conflicts involving more than two aircraft, at least two maneuvers were usually required. Another tool, a visual presentation of relative position vectors, has shown similar benefits as our CAT tool (Vuckovic et al, 2013). Indeed, the new display tool also showed better performance only in the most complex scenarios as well as in the present study. Besides, it has also been shown that the reliability of an automated decision aid could influence controllers' performance (Metzger and Parasuraman, 2005). In particular, when the automation was imperfect, the conflict detection was better under manual conditions than under automated conditions. Thus, if the $C A T$ tool had to be used in operational settings, it should also be previously evaluated in unreliable conditions, i.e., when the uncertainties model used in the trajectory prediction does not match aircraft final trajectories.

Nevertheless, before introducing an automated system in operational settings, one has to determine sensitive scenarios of unsafe situations, as both humans and machines can be unreliable. Feasibility studies in ATC should at least assess the impact on three elements: operator's workload, safety criteria and perceived unreliability (Vanderhaegen, 1999).

The present CAT tool was not designed to give a solution or to automatically solve the conflicts, but only to help controllers finding an optimal solution. This might essentially be useful during training. Further studies are planned to assess the potential benefit of using the CAT tool during initial ATCO training. 


\section{References}

Alaeddini A, Erzberger H, Dunbar W (2011) Distributed logic-based conflict resolution of multiple aircraft in planar en-route flight. In: AIAA Guidance, Navigation and Control Conference and Exhibit

Allignol C, Barnier N, Durand N, Alliot JM (2013) A new framework for solving en-routes conflicts. In: 10th USA/Europe Air Traffic Management Research and Developpment Seminar

Alliot JM, Bosc JF (1996) Air Traffic Control, chess playing and chess programs : lessons to learn. In: ICTAI 1996, 8th International Conference on Tools for Artificial Intelligence, Toulouse, France, URL https://hal-enac. archives-ouvertes.fr/hal-01021665

Arthur WC, McLaughlin MP (1998) User request evaluation tool (uret). interfacility conflict probe performance assessment. In: Procceedings of the 2nd USA/Europe ATM R and D Seminar

Baddeley AD, Logie RH (1999) Working memory: The multiple-component model. In: Miyake A, Shah P (eds) Models of Working Memory, Cambridge University Press, pp 28-61, DOI 10.1017/cbo9781139174909.005, URL https : //doi . org $/ 10.1017 \% 2 \mathrm{Fcbo9781139174909.005}$

Bakker G, Blom H (2000) Wp1: Comparative analysis of probabilistic conflict prediction approaches in atm. Tech. rep., NLR Contract Report

Bisseret A (1971) Analysis of mental processes involved in air traffic control. Ergonomics 14(5):565-570, DOI 10.1080/00140137108931276, URL https:// doi.org/10.1080\%2F00140137108931276

Bisseret A (1981) Application of signal detection theory to decision making in supervisory control the effect of the operator's experience. Ergonomics 24(2):81-94

Borst C, Bijsterbosch VA, van Paassen MM, Mulder M (2017) Ecological interface design: supporting fault diagnosis of automated advice in a supervisory air traffic control task. Cognition, Technology \& Work 19(4):545-560, DOI 10.1007/ s10111-017-0438-y, URL https : //doi .org/10.1007/s10111-017-0438-y

Christodoulou MA, Kontogeorgou C (2008) Collision avoidance in commercial aircraft free flight via neural networks and non-linear programming. Int J Neural Syst 18(5):371-387

Corver S, Grote G (2016) Uncertainty management in enroute air traffic control: a field study exploring controller strategies and requirements for automation. Cognition, Technology \& Work 18(3):541-565, DOI 10.1007/s10111-016-0373-3, URL https://doi.org/10.1007/s10111-016-0373-3

Dean G, Fron X, Miller W, Nicolaon J (1995) Arc2000 : An investigation into the feasibility of automatic conflict. Tech. rep., Centre Experimental Eurocontrol

Duong VN, Hoffman E, Nicolaon JP (1997) Initial results of investigation into autonomous aircraft concept (freer-1). In: Procceedings of the 1st USA/Europe ATM $\mathrm{R}$ and $\mathrm{D}$ Seminar

Durand N (1996) Optimisation de trajectoires pour la résolution de conflits en route. $\mathrm{PhD}$ thesis, INPT

Durand N, Granger G (2003) A traffic complexity approach through cluster analysis. In: 5th ATM R\&D Seminar 
Durand N, Alliot JM, Noailles J (1996) Automatic aircraft conflict resolution using genetic algorithms. In: Proceedings of the Symposium on Applied Computing, Philadelphia, ACM

Durand N, Gotteland JB, Matton N (2018) Visualizing complexities: the human limits of air traffic control. Cognition, Technology and Work DOI 10.1007/s10111-018-0468-0, URL https://hal-enac . archives-ouvertes . fr/hal-01707751

Edwards T, Homola J, Mercer J, Claudatos L (2017) Multifactor interactions and the air traffic controller: the interaction of situation awareness and workload in association with automation. Cognition, Technology \& Work 19(4):687698, DOI 10.1007/s10111-017-0445-z, URL https://doi.org/10.1007/ s10111-017-0445-z

Erzberger H (1997) Conflict probing and resolution in the presence of errors. In: Procceedings of the 1st USA/Europe ATM R and D Seminar

Frazzoli E, Mao ZH, Oh JH, Feron E (2001) Resolution of conflicts involving many aircraft via semidefinite programming. AIAA Journal of Guidance, Control and Dynamics 24(1)

Gariel M, Feron E (2009) 3d conflict avoidance under uncertainties. In: Digital Avionics Systems Conference, 2009. DASC '09. IEEE/AIAA 28th, pp 4.E.3-14.E.3-8, DOI 10.1109/DASC.2009.5347480

Gentner D (2014) Mental Models. Psychology Press, DOI 10.4324/9781315802725, URL https : //doi .org/10.4324\%2F9781315802725

Gobet F, Charness N (2006) Expertise in chess. In: The Cambridge Handbook of Expertise and Expert Performance, Cambridge University Press, pp 597615, DOI 10.1017/9781316480748.031, URL https://doi.org/10.1017\% 2F9781316480748.031

Juricic B, Varesak I, Bozic D (2011) The role of the simulation devices in air traffic controller training. In: 19th international symposium on electronics in transports (ISEP)

Karikawa D, Aoyama H, Takahashi M, Furuta K, Wakabayashi T, Kitamura M (2013) A visualization tool of en route air traffic control tasks for describing controller's proactive management of traffic situations. Cognition, Technology \& Work 15(2):207-218, DOI 10.1007/s10111-012-0222-y, URL https://doi. org/10.1007/s10111-012-0222-y

Karikawa D, Aoyama H, Takahashi M, Furuta K, Ishibashi A, Kitamura M (2014) Analysis of the performance characteristics of controllers' strategies in en route air traffic control tasks. Cognition, Technology \& Work 16(3):389-403, DOI 10.1007/ s10111-013-0268-5, URL https ://doi.org/10.1007/s10111-013-0268-5

Meckiff DC, Gibbs DP (1994) PHARE : Highly interactive problem solver. Tech. rep., Eurocontrol

Metzger U, Parasuraman R (2005) Automation in future air traffic management: Effects of decision aid reliability on controller performance and mental workload. Human Factors: The Journal of the Human Factors and Ergonomics Society 47(1):35-49, DOI 10.1518/0018720053653802, URL https://doi.org/ $10.1518 \% 2 \mathrm{~F} 0018720053653802$ 
Myles-Worsley M, Johnston WA, Simons MA (1988) The influence of expertise on $\mathrm{x}$-ray image processing. Journal of Experimental Psychology: Learning, Memory, and Cognition 14(3):553-557, DOI 10.1037/0278-7393.14.3.553, URL https: //doi.org/10.1037\%2F0278-7393.14.3.553

Neal A, Kwantes PJ (2009) An evidence accumulation model for conflict detection performance in a simulated air traffic control task. Human Factors: The Journal of the Human Factors and Ergonomics Society 51(2):164-180, DOI 10.1177/ 0018720809335071, URL https : //doi.org/10.1177\%2F0018720809335071

Oh JH, Shewchun J, Feron E (1997) Design and analysis of conflict resolution algorithms via positive semidefinite programming [aircraft conflict resolution]. In: Decision and Control, 1997., Proceedings of the 36th IEEE Conference on, vol 5, pp 4179-4185 vol.5, DOI 10.1109/CDC.1997.649489

Pallottino L, Feron E, Bicchi A (2002) Conflict resolution problems for air traffic management systems solved with mixed integer programming. Intelligent Transportation Systems, IEEE Transactions on 3(1):3-11, DOI 10.1109/6979.994791

Prevot T, Lee P, Smith N, Palmer E (2005) Atc technologies for controller-managed and autonomous flight operations. In: AIAA Guidance, Navigation and Control Conference and Exhibit

Prevot T, Homola J, Mercer J (2008) Initial study of controller/automation integration for nextgen separation assurance. In: AIAA Guidance, Navigation and Control Conference and Exhibit

Prevot T, Homola J, Martin L, Mercer J (2011) Automated air traffic control operations with weather and time-constraints. In: 9th ATM R\&D Seminar

Price A, Meckiff C (1997) Hips and its application to oceanic control. In: 1st ATM R\&D Seminar

R Core Team (2018) R: A Language and Environment for Statistical Computing. R Foundation for Statistical Computing, Vienna, Austria, URL https://www . R-project.org/

Rantanen EM, Nunes A (2005) Hierarchical conflict detection in air traffic control. The International Journal of Aviation Psychology 15(4):339362, DOI 10.1207/s15327108ijap1504_3, URL https://doi.org/10.1207\% 2Fs15327108ijap1504_3

Seamster TL, Redding RE, Cannon JR, Ryder JM, Purcell JA (1993) Cognitive task analysis of expertise in air traffic control. The International Journal of Aviation Psychology 3(4):257-283, DOI 10.1207/s15327108ijap0304_2, URL https:// doi.org/10.1207\%2Fs15327108ijap0304_2

Tabachneck-Schijf HJ, Leonardo AM, Simon HA (1997) CaMeRa: A computational model of multiple representations. Cognitive Science 21(3):305350, DOI 10.1207/s15516709cog2103_3, URL https://doi.org/10.1207\% 2Fs15516709 cog2103_3

Trapsilawati F, Wickens CD, Qu X, Chen CH (2016) Benefits of imperfect conflict resolution advisory aids for future air traffic control. Human Factors: The Journal of the Human Factors and Ergonomics Society 58(7):1007-1019, DOI 10.1177/ 0018720816655941, URL https://doi .org/10.1177\%2F0018720816655941

Tversky B (2005) Functional significance of visuospatial representations. In: Shah P, Miyake A (eds) The Cambridge Handbook of Visuospatial Thinking, Cambridge 
University Press, pp 1-34, DOI 10.1017/cbo9780511610448.002, URL https: //doi.org/10.1017\%2Fcbo9780511610448.002

Vanderhaegen F (1999) Toward a model of unreliability to study error prevention supports. Interacting with Computers 11(5):575-595, DOI 10.1016/ s0953-5438(98)00044-7, URL https://doi.org/10.1016\%2Fs0953-5438\% $2898 \% 2900044-7$

Vuckovic A, Sanderson P, Neal A, Gaukrodger S, Wong BLW (2013) Relative position vectors. Human Factors: The Journal of the Human Factors and Ergonomics Society 55(5):946-964, DOI 10.1177/0018720813481803, URL https ://doi. org $/ 10.1177 \% 2 \mathrm{~F} 0018720813481803$ 